\title{
New and Extended Appliteations for USP Drug Release Apparatus 3.
}

1"Ian Borst, 2Sydney Ugwu and 3A. H. Beckett

1 Drug Testing Laboratory, Laboratory Services Branch, Ministry of Health, Ontario, Canada.

2 VanKel Industries, Inc. R\&D Laboratory, Edison, N7, USA

3 Professor Emeritus, University of London, U.K.

* To whom correspondence should be addressed

\section{Abstract}

The bistory of the development and the principles of the operation of USP 3

are described briefly. Its use for the dissolution testing of immediate release

products as well as flexibility in the examination and design of controlled

release is summarized. Special emphasis is given to the advantages of the

instrument in mimicking the changes in physicochemical conditions and

mechanical forces experienced by products in the G.I. tract.

\section{Background}

1

he concept of USP apparatus 3 resulted from a presentation at the 1980 Federation Internationale

Pharmaceutique (F.I.P) conference. This presentation discussed the problem that dissolution results obtained with USP Apparatus 1 and 2 may be affected significantly by shaft wobble, location, centering, deformation of baskets and paddles as well as the presence of bubbles in the dissolution medium etc. It was agreed at the conference that major problems could arise in the acceptance of pharmaceutical products in international trade due to the variations in dissolution data caused by physical, mechanical and system hydrodynamics inherent in Apparatuses 1 and 2 which were not a reflection of variations in product quality .

In the 70's, Professor Beckett and many workers in the field were using the rotating bottle method (NF XII 1965 - XIV 1975) for the evaluation of controlled release products, especially those in the pellet form. The rotating bottle method provided a sound hydrodynamic system i.e. the dosage form moved through the dissolution medium freely as the bottle rotated, which is in contrast to the movement of media relative to the dosage form in USP 1 and 2 in which various portions of the bulk medium move at different rates. Despite the proven $\mathrm{pH}$ profiling capability and highly reproducible dissolution profiles using the rotating bottle method, there were some shortcoming features such as being labor intensive and difficult to fully automate media changes, which were also the reasons for the method not obtaining its official status in the USP.

With the research effort over the years, it was becoming increasingly apparent that to approach the goal of in vitro - in vivo correlation, the ability to sequentially alter the: $\mathrm{pH}$, molarity, anions, cations, viscosity, buffers, surface active agents and degree of agitation during the dissolution process was essential.

The recognition of the above factors prompted the development of the BIO-DIS by a team of scientists working under Beckett's direction in London, UK. The design of the BIO-DIS incorporated the hydrodynamic features from the rotating bottle with the capability of full automation of media and agitation changes and flexibility to interface with either UV/VIS or HPLC for on-line real time complete analysis.

The USP Apparatus 3 (Figure 1) consists of a heated bath containing rows of outer tubes (usually 6) which contain the media, while in each row there are 6 inner tubes (newer units have an additional 7th tube for standard solution) containing the product to be analyzed. Operation involves programming the agitation rate, dips per minute (dpm), of the up and down movement for the inner tube

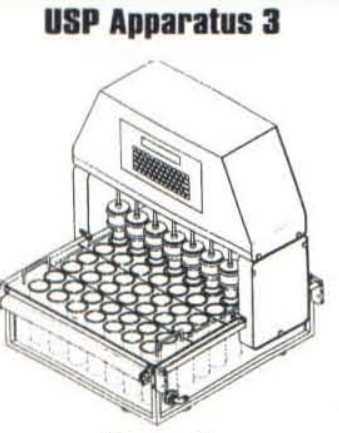

Figure 1. inside the outer one. The inner tube consists of a glass cylinder threaded on both ends, end caps which screw onto the cylinder end screens which retain samples during operation as indicated in USP 23. The outer tube containing the appropriate medium is in contact with the water bath to allow the medium to be maintained at $37^{\circ} \mathrm{C}$. At the

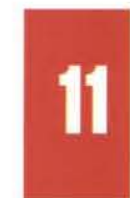




\section{USP Apparatus 3....Eont.}

base and top of the inner tube there is a soft mesh and the product contained in the inner tube becomes suspended in the moving media during the reciprocating action. On the up stroke, the bottom mesh of the inner tube moves upward to contact the product and on the down stroke the product leaves the mesh and floats freely within the inner tube. Thus the action produced carries the product being tested through a moving medium.

\section{Principles of Dperation}

At the start of the test, the inner tubes as programmed, descend slowly into the outer tubes of the first row containing the dissolution medium and the reciprocating motion starts. At the end of the time allocated for this row, the inner tubes with their product content rise above the outer tubes, drain for the programmed time, and then are automatically moved to above the second row of the outer tubes into which they slowly descend and commence their reciprocating action. Similar programmed events occur for the subsequent rows of outer tubes.

Each of the outer tubes are filled with $250 \mathrm{~mL}$ of medium. As there are six (6) rows of outer tubes the volume of medium that can be used in a single dissolution test on each unit dosage can be $6 \times 250$ $\mathrm{mL}=1500 \mathrm{~mL}$. If sink conditions cannot be achieved with the above volume then additional fresh media in multiples of $250 \mathrm{~mL}$ can be used by replacement of media from previous rows which increases the maximum volume of media necessary for a particularly slow release dosage form. This is possible because the system can be programmed to continue operation by returning to the first row and repeating the entire cycle with fresh media.

USP 3 has the capability to provide numerous programmable options in time, agitation speed and media changes, which are necessary during dissolution methodology development of a product to study in vitro - in vivo correlations. However, once the desired dissolution conditions have been established, a simplified program may be written for quality control purposes utilizing the key dissolution parameters to characterize the dissolution release of the dosage form.

\section{Geometry and Deaeration}

Small modifications in geometry of the dissolution vessel and the formation of air bubbles which usually form in nondearated media change the flow characteristics of media in conventional appara- tuses like USP 1 and 2, and can result in erroneous dissolution rates. Due to the design and reciprocating pump action of the USP Apparatus 3 assembly, dissolution rates are not affected by changes in the geometry or the presence of air bubbles. The USP Apparatus 3 assembly has been studied to assess the effect of the geometry of the outer tube or glass vessel and the need for deaeration.

The influence of the outer tube capacity and geometry was studied by determining the dissolution profiles of USP salicylic acid $300 \mathrm{mg}$ tablets and FDA prednisone $10 \mathrm{mg}$ calibrator tablets as shown in Figures 2 \& 3 . The results show that changes

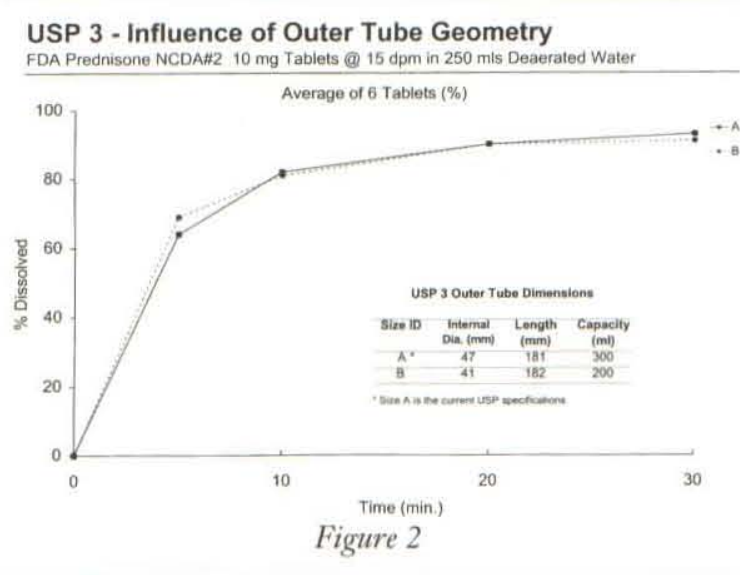

USP 3 - Influence of Outer Tube Geometry USP Salicylic Acid $300 \mathrm{mg}$ Tabiets @ 15 dpm in 250 mis Deaerated Buffer pH 7.4

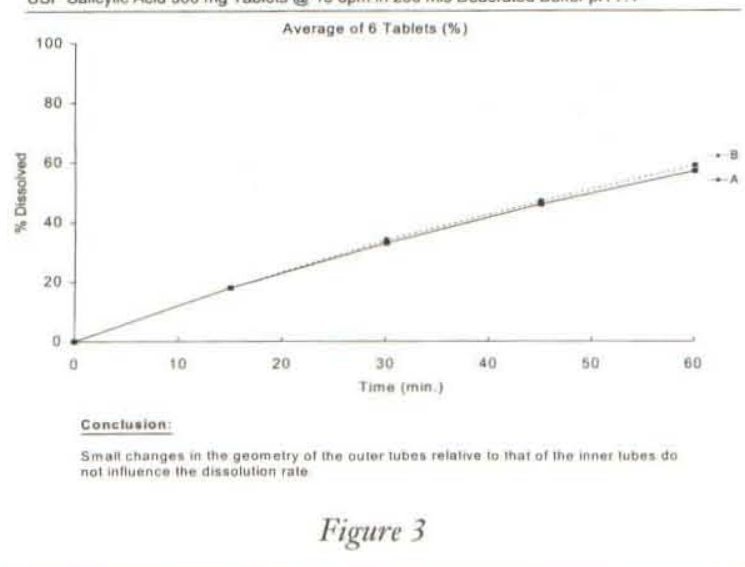

within the range indicated in the geometry and capacity of the outer tube relative to the inner tube do not influence the dissolution rate. Also Figures 4 of 5 employing the same calibrator tablets show that deaeration has negligible effect on the dissolution rates because the absence or presence of bubbles does not alter the movement of the dosage form 

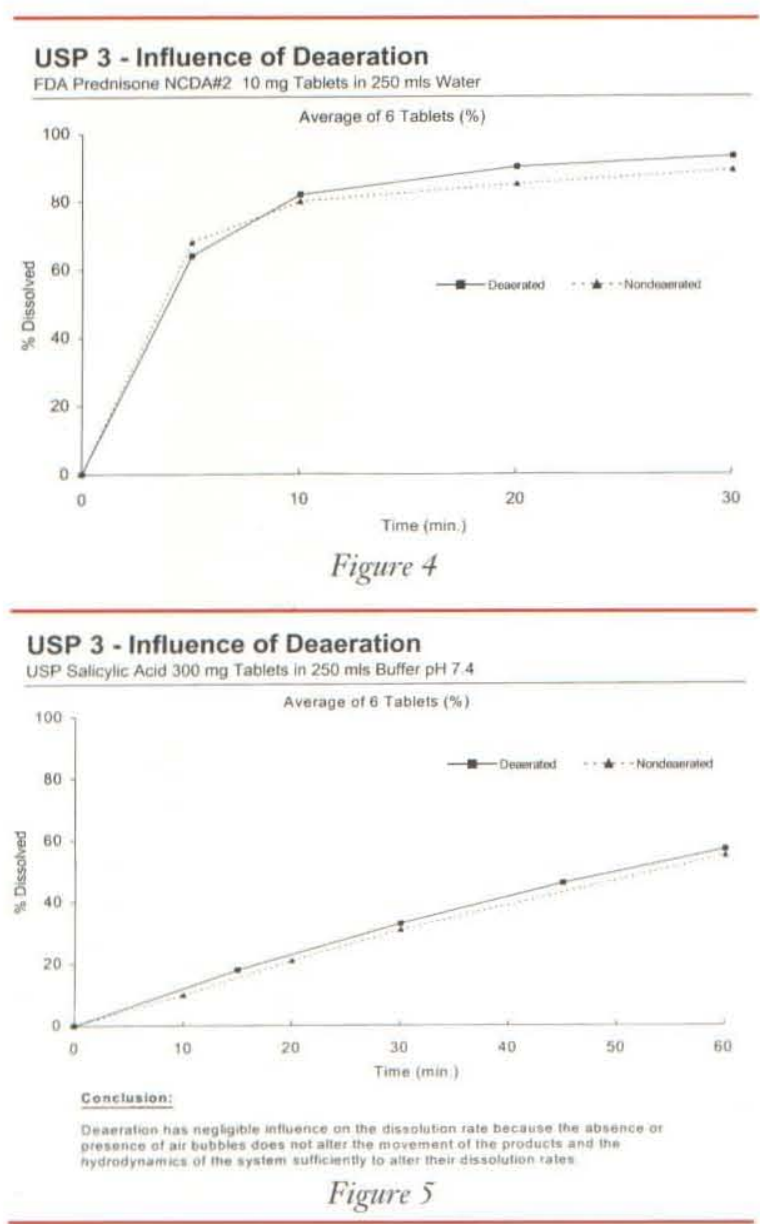

and the hydrodynamics of the system sufficiently to affect their dissolution rates.

The apparatus has been extensively studied in Europe and published data indicate good correlation with the rotating bottle apparatus and impressive correlations with bioavailability data $(1,2)$. The early papers covered the BIO-DIS operation and validation by Graepel H. P. et al. in Europe (1) and Esbelin B. et al. in America (3). Later papers included Sanghvi et al. (4), Borst (5) and McCall et al. (6). More recently Farrell et al. in Irelend (7) has shown that USP 3 is a better discriminator than USP 2 to predict the in-vivo impact of changes in formulation variables for a hydrophilic matrix long acting tablet. The American data base is limited but has shown increasingly considerable interest to date, especially with the development of more controlled release products. The apparatus was evaluated at the 1990 USP open conference in regard to an alternative dissolution apparatus and was made official in USP 22, fourth supplement. The results of a collaborative study of USP drug release calibrators for Apparatus 3 was published in Pharmacopeial Forum (Jan-Feb. 1994). Subsequently, the apparatus 3 suitability test of Theophylline ER (multiple unit) and Chlorpheniramine ER tablets (single unit) appeared in USP 23 first supplement, which established official calibrators for USP 3.

\section{Applications to Dissolution of Controlied and Immediate Release Products. \\ 1 - Controlled Release Products \\ This has been discussed above.}

\section{2 - Immediate Release Products}

At a recent AAPS/USP workshop in Arlington, VA (8), Beckett addressed and focused on the full potential application of the BIO-DIS for both controlled and immediate release products. Although this apparatus is well known and mainly used for evaluation of controlled release products, many involved in dissolution testing have not realized its value in testing of immediate release products relative to the use of other methods. USP Apparatus 1 (basket) has several serious disadvantages such as tendency for gummy substances clogging the basket screen, extremely sensitive to dissolved gases in the dissolution fluid, inadequate flow characteristics when particles containing drug leave the basket and float to the surface if they are of low density or settle on the base of the vessel, if they are denser, where they do not move in the medium. These effects have significant impact on the observed dissolution rate of solid dosage forms. USP Apparatus 2 (paddle) overcomes many disadvantages of the basket. The modern designed instruments virtually eliminate the mechanical and physical problems previously reported, e.g., centering, vibration etc. However, the "coning" phenomena on USP 2 is commonly observed and reported due to the system hydrodynamics especially when the instrument is operated at $50 \mathrm{rpm}$ and the products contain a sparingly soluble drug in a high percentage of insoluble excipients. The formation of the cone significantly reduces the dissolution rate and produces a wide variation in results, which affects the uniformity of data and therefore meaningful product specifications. This poorly stirred cone exists even if particulate matter is not present to make it visible. Thus if tablets are placed at the base of the 


\section{USP Apparatus 3....cont.}

vessel, the dissolution results can be different than if placed to the side of this base. The size and shape of sinkers and fixed sampling probes can perturb this non-visible cone and thus lead to erratic results.

With earlier lower cap designs, the applications of USP 3 for the testing of immediate release products had some concerns because of the inadequate

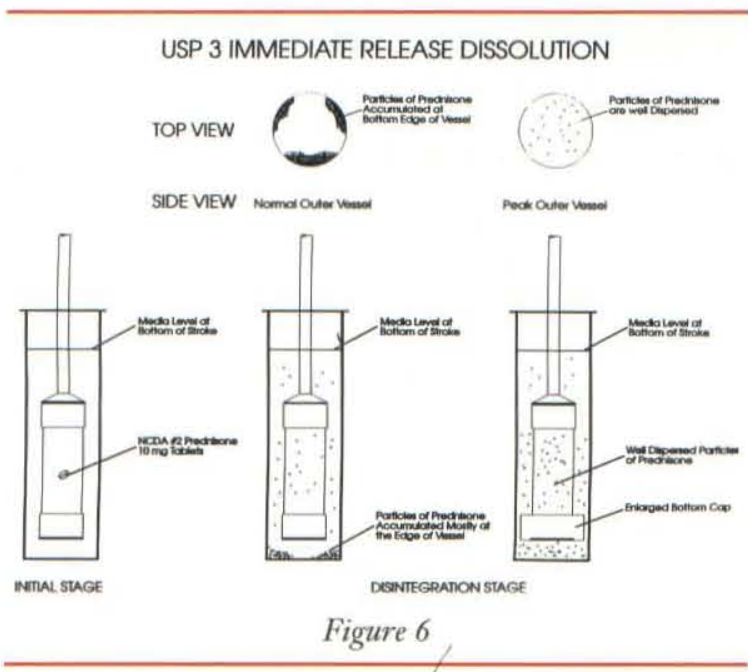

agitation of particles which pass through the lower mesh and go to the base of the outer tube. The development of a specially designed and fabricated VanKel Type wider lower cap (Figure 6) overcomes the probelm to give excellent dispersion of the particles. The following experimental results show the potential of USP Apparatus 3 for testing of imme-

Comparison of Dissolution for NCDA Prednisone $10 \mathrm{mg}$ Tablets Using Wide and Regular Caps in USP 3

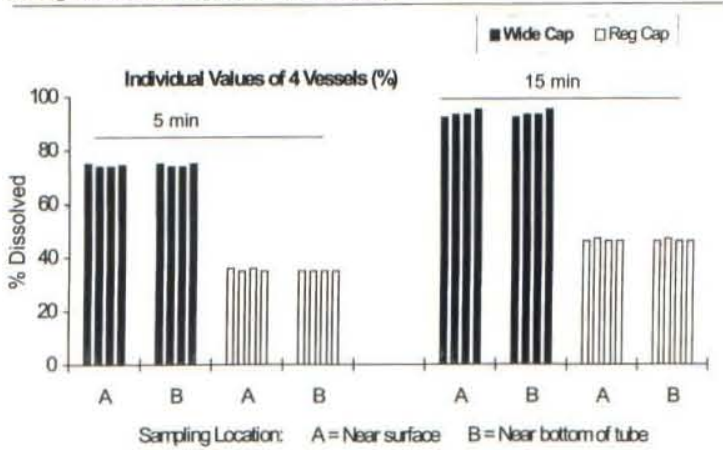

Figure 7

diate release products. Dissolution of the NCDA Prednisone $10 \mathrm{mg}$ calibrator from the FDA was conducted in USP 3 using water as the medium at $15 \mathrm{dpm}$ for 30 minutes. The results, as expected, indi- cate a significant increase in the rate of dissolution (Figure 7) using the new wider diameter cap because the particles containing drug which come through the mesh are now moved throughout the medium. The greater agitation in the outer tube is due to the piston action of the new cap configuration which gives correct mixing in the outer tube. Dissolution samples taken and analyzed from near the surface and bottom of the dissolution tube respectively to assess the uniformity of mixing show that similar drug concentrations are found at both locations. Tolbutamide tablets (500 mg strength) are another example (Figure 8 ), showing uniform mixing of the formulation particles with the wide cap, while with the regular cap there is

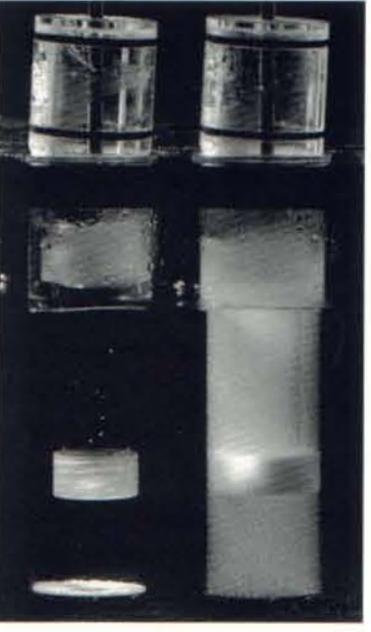

Figure 8

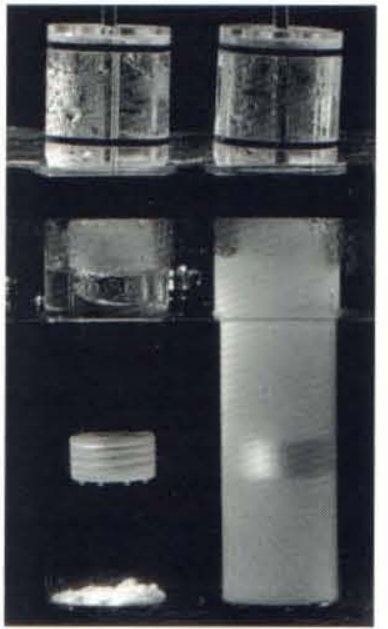

Figure 9 only limited mixing of the solid particles which have come through the mesh. A simple physical demonstration of the difference in the movement of particles in the outer tube, at $15 \mathrm{dpm}$, using the regular and the wider cap can be seen by adding the same amount of microcrystalline cellulose to the inner tubes (Figure 9) to pass through the mesh into the outer tubes.

Also, similar dissolution rates to USP 3 as shown above were obtained for NCDA Prednisone $10 \mathrm{mg}$ tablets using USP 2 with PEAK vessels (9) at rotation speeds of $75 \mathrm{rpm}$ and $100 \mathrm{rpm}$, respectively, which are compared in Figure 10. Thus, this provides more opportunity for the design of dissolution tests which will give comparable results by USP 3 or USP 2 using PEAK vessels. 
Comparison of USP 3 with USP 2 Using PEAK Vessels for Prednisone $10 \mathrm{mg}$ Tablets (NCDA)

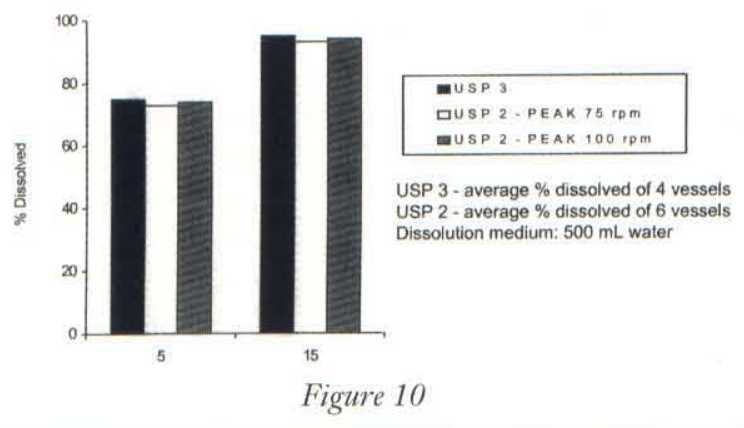

For sparingly soluble immediate release products that disintegrate, dissolution should be conducted at $\mathrm{pH} 1.5$ and the test could be repeated at $\mathrm{pH} 6.9$ for drugs that are more soluble at the higher $\mathrm{pH}$ values, provided the drug possesses sink solubility conditions at the appropriate $\mathrm{pH}$ and volume $(250 \mathrm{~mL})$.

3 - In-vitro Predictor of Bioavailability in the Fed and Fasted States

When performing a dissolution test on controlled or extended release products, the outer tubes of the first row should contain media of acidic $\mathrm{pH}$, i.e. 1.5 , in order to simulate gastric conditions. The outer tubes of the second row contain media of $\mathrm{pH}$ 4.5 with or without surfactants to represent conditions of the upper portion of the small intestine, followed by a third row of $\mathrm{pH} 6.9$ to represent the conditions of the lower small intestine. The fourth row would contain media of $\mathrm{pH} 7.2$, followed by a fifth row with media of $\mathrm{pH} 7.5$, and a sixth row also containing media of $\mathrm{pH} 7.5$. If a simulation of the FASTED state is desired it would involve dipping of the product in the first row for 1 hour. For a simulation of the FED state, a dipping time of 4 hours in the first row, followed by 1 hour dipping time in the second row should be employed. The appropriate dipping times in the following rows would be determined based upon whether a 12 or 24 hour product is being analyzed.

The dip speed for each row should be 10 or 15 dips per minute (dpm), with the exception for simulation of the fed state (first row $\mathrm{pH} 1.5$ for 4 hours) when the dipping rate should be increased to 30 or $40 \mathrm{dpm}$ to represent the greater churning and turbulence in the stomach in the fed state; inert beads of mixed density can be added to represent interaction with moving solid particles of food. Katori et al. in
Japan (10) also recommends development of invitro release systems that take into account GI factors which include GI residence time, mechanical destructive forces in the gut and agitation rate. Katori has shown that destructive mechanical forces in the GI tract play an important role in affecting the drug release from an erodable single unit CR dosage form (11) Thus, when the in-vivo results in fed state biostudies show products to be bioinequivalent and the dissolution results by USP 1 or 2 show no differences, USP 3 may be the method of choice using the in-vitro modifications described above to simulate the fed state. This assessment has been supported recently by Farrell et al. (7) showing that biostudies conducted in the fed state on a CR dosage form could be correlated with USP 3 , but not dissolution data using USP 2.

\section{Conclusion}

USP 3 should be considered the first line apparatus in product development of controlled release preparations, because of its usefulness and convenience in exposing products to mechanical as well as a variety of physicochemical conditions which may influence the release of products in the GI tract. The apparatus is now fully automated and this model is undergoing evaluation to facilitate work in quality control as well as in R \& D laboratories.

The new VanKel type wider caps for the inner tubes as an alternative to the regular caps allows USP 3 to provide a viable alternative to USP 1 and 2 for immediate release products.

\section{Acknowledgments}

The authors thank both Angelita Reyes and Tinh Quach for their analytical assistance in the preparation of the manuscript. Also, Michael Pocelinko of VanKel Industries for review of the manuscript and the Instructional Media Centre, Laboratory Services Branch, Ontario Ministry of Health for presentation of photographs.

\section{References:}

(1) Graepel H.P., Momberger, H. and Kubn D., Pharm.

Ztg. 1986; 131 (31); 182001823.

(2) Hadzja B.W., Pharm Res 1986; (suppl), $39 \mathrm{~S}$

(3) Esbelin B., Beyssac E., Aiace 7-M., Shiu G. Kand

Skelly, 7.P., 7. Pharm Sci., 1991;80(10) 991-994

See References, USP Apparatus 3 continued on page 18 
References USP Apparatus 3 continued from page 13

(4) Sangbvi P.P., Nambiar, 7.S. et al., Drug Dev. Ind Pharm.1994; 20(6); 961-980.

(5) Borst, S.I., Drug Dev. Ind Pharm. 1991; 17(17), 3332339.

(6) McCall T.W., et al., TIMERx Technologies 1995 Proceedings Abstract 1115 (22nd International symposium on Controlled Release of Bioactive Materials.

(7) AAPS International Meeting, Seattle, Washington, Oct. 27-31, 1996, Farrell, C., Kinaban, A., Fogarty,F., et al., Poster \#'s PDD 7210, PDD 7211 \& PDD 7212.

(8) AAPS/USP Workshop, Arlington, VA, April 18-19, 1996.

(9) Beckett, A. H., Quach, T. T. \& Kurs, G., Dissolution Tecbnologies, 1996, Vol. 3, Issue 2, Page 7.

(10) Katori, N., Ma, W, Aoyagi, N. \& Kojima, S., Pbarm. Res. 1996,13, (10), 1541-1546.

(11) Shameem, M., Katori, N., Aoyagi, N. \& Kojima, Pharm. Res.1995, 12, 1049-1054. 